\title{
Large-eddy Simulation of Realistic Gas Turbine Combustors
}

\author{
Moin, P. , \& Apte, S. V. ${ }^{\dagger}$ \\ Center for Integrated Turbulence Simulations \\ Stanford University, Stanford, CA 94305-3030 \\ http://cits.stanford.edu/
}

December 1, 2005

\begin{abstract}
Large-eddy simulation (LES) is a promising technique for accurate prediction of reacting multiphase flows in practical gas-turbine combustion chambers involving complex physical phenomena of turbulent mixing and combustion dynamics. This paper discusses development of advanced models for liquid fuel atomization, droplet evaporation, droplet deformation \& drag, and turbulent combustion specifically for gas-turbine applications. The non-dissipative, yet robust numerical scheme for arbitrary shaped unstructured grids developed by Mahesh et al. [1] is modified to account for density variations due to chemical reactions. A systematic validation and verification study of the individual spray models and the numerical scheme is performed in canonical and complex combustor geometries. Finally, a multi-scale, multi-physics, turbulent reacting flow simulation in a real gas-turbine combustor is performed to assess the predictive capability of the solver.
\end{abstract}

\footnotetext{
*Franklin and Caroline Johnson Professor of Mechanical Engineering, Associate Fellow, AIAA, moin@stanford.edu

${ }^{\dagger}$ Research Associate, Department of Mechanical Engineering. Presently at Oregon State University, sva@engr.orst.edu
} 


\section{Introduction}

The combustion chambers of gas-turbine based propulsion systems involve complex phenomena such as atomization of liquid fuel jets, evaporation, collision/coalescence of droplets, and turbulent mixing of fuel and oxidizer giving rise to spray-flames. Accurate observations and quantitative measurements of these processes in realistic configurations are difficult and expensive. Better understanding of these flows for design modifications, improvements, and exploring fundamental physics demands high-fidelity numerical studies in realistic configurations. Specifically, good predictive capability for swirling, highly turbulent reacting flows in complex geometries is necessary.

To date the engineering prediction of such flows in realistic configurations has relied predominantly on the Reynolds-averaged Navier-Stokes equations (RANS) [2, 3]. In RANS, turbulence models for the Reynolds stress tensor provide time (or ensemble) -averaged solutions to the Navier-Stokes equations. Though computationally efficient, RANS-based models for two-phase reacting flows do not represent the relevant flow quantities accurately even in simple configurations. LES and direct numerical simulation (DNS) techniques have been shown to give good predictions of turbulent flows in simple configurations [4]. Recently, Pierce \& Moin [5] have shown the superiority of LES to RANS in accurately predicting turbulent mixing and combustion dynamics in a coaxial combustor geometry. Kim \& Syed [6] and Mongia [7] provide a detailed overview on the importance and role of LES in designing advanced gas-turbine combustors. The flowfield inside the combustor is highly swirling, separated and turbulent with complex features such as mixing of secondary cooling air with hot combustion products. The spray flame is stabilized by the recirculation bubble created by swirling flow. Multiple, turbulent jets in cross flow play an important role in scalar mixing; may influence pollutant formation, and elimination of any 'hot-spots' in the combustor exit. LES is considered very attractive in predicting these flow features and their sensitivity to design modifications. However, presently LES has largely been used to investigate flows in simple configurations. Our goal is to extend the LES methodology to realistic geometries involving complex physics of multiphase, reacting 
flows.

In LES, three-dimensional, unsteady Navier-Stokes equations are spatially filtered, the large scales are computed directly, and only the effect of unresolved subgrid scales is modeled. The penalty is increased computational cost. In addition, the numerical algorithms used for LES must be energy conserving and strictly non-dissipative, as numerical dissipation has been shown to be detrimental in accurate prediction of turbulent flows [8]. Furthermore, the complex geometry of practical combustors necessitates use of unstructured grids due to the flexibility they offer in handling complex configurations as well as significant savings in the number of control volumes as compared to the body-fitted structured grids.

Recently, Mahesh et. al. [1] have developed a new numerical method with the characteristics necessary for simultaneously accurate and robust LES on unstructured grids. These competing ends were achieved by developing a method around the principle of discrete kinetic energy conservation with no artificial dissipation. Based on this numerical scheme, a parallel, arbitrary elements, unstructured grid, finite-volume code has been developed specifically to perform LES of complex combustor geometries. The solver is named after late Charles David Pierce (1969-2002) who made several lasting contributions to the LES of reacting flows. The original incompressible formulation by Mahesh et al. [1] is extended to simulate variable density, low-Mach number flows with integrated models for turbulent combustion and spray dynamics $[9,10,11]$. In order to trust the numerics and know the limitations of the models used, a systematic validation and verification study evaluating their predictive capability is of utmost importance. Detailed experiments independently addressing droplet dispersion, droplet evaporation, breakup, and turbulent combustion (with gaseous fuel) have been performed, however, experiments involving multiphase reacting flows in simplified combustors are needed to further advance the models for spray dynamics and turbulent combustion.

In the following sections, the mathematical formulation for gas and liquid phases, advanced subgrid models for droplet breakup, evaporation, deformation, and drag are described. Results from numerous validation studies performed are reported. Ongoing efforts to further develop advanced physical models and numerical algorithms for improved speedup are summarized. 


\section{Mathematical Formulation}

We solve the variable density, low-Mach number, Navier-Stokes equations for the gas-phase. The formulation is based on flamelet progress-variable (FPV) approach developed by Pierce \& Moin [5] for LES of non-premixed, turbulent combustion. The liquid phase is treated in the Lagrangian framework with efficient particle tracking scheme on unstructured grids, which allows simulation of millions of independent droplet trajectories. A summary of the filtered Eulerian/Lagrangian equations and subgrid models for unclosed terms and droplet dynamics is given below.

\subsection{Filtered LES Equations for Gas-Phase}

The gas phase continuity, scalar, and momentum equations are,

$$
\begin{gathered}
\frac{\partial\left(\bar{\rho}_{g} \tilde{u}_{j}\right)}{\partial x_{j}}=-\frac{\partial \bar{\rho}_{g}}{\partial t}+\overline{\dot{S}}_{m} \\
\frac{\partial\left(\bar{\rho}_{g} \tilde{Z}\right)}{\partial t}+\frac{\partial\left(\bar{\rho}_{g} \tilde{Z} \tilde{u}_{j}\right)}{\partial x_{j}}=\frac{\partial}{\partial x_{j}}\left(\bar{\rho}_{g} \tilde{\alpha}_{Z} \frac{\partial \tilde{Z}}{\partial x_{j}}\right)-\frac{\partial q_{Z j}}{\partial x_{j}}+\overline{\dot{S}}_{Z} \\
\frac{\partial\left(\bar{\rho}_{g} \tilde{C}\right)}{\partial t}+\frac{\partial\left(\bar{\rho}_{g} \tilde{C} \tilde{u}_{j}\right)}{\partial x_{j}}=\frac{\partial}{\partial x_{j}}\left(\bar{\rho}_{g} \tilde{\alpha}_{C} \frac{\partial \tilde{C}}{\partial x_{j}}\right)-\frac{\partial q_{C j}}{\partial x_{j}}+\overline{\dot{\omega}}_{C} \\
\frac{\partial\left(\bar{\rho}_{g} \tilde{u}_{i}\right)}{\partial t}+\frac{\partial\left(\bar{\rho}_{g} \tilde{u}_{i} \tilde{u}_{j}\right)}{\partial x_{j}}=-\frac{\partial \bar{p}}{\partial x_{i}}+\frac{\partial\left(2 \bar{\mu} \tilde{S}_{i j}\right)}{\partial x_{j}}-\frac{\partial q_{i j}}{\partial x_{j}}+\overline{\dot{S}}_{i}
\end{gathered}
$$

where

$$
\tilde{S}_{i j}=\frac{1}{2}\left(\frac{\partial \tilde{u}_{i}}{\partial u_{j}}+\frac{\partial \tilde{u}_{j}}{\partial u_{i}}\right)-\frac{1}{3} \delta_{i j} \frac{\partial \tilde{u}_{k}}{\partial x_{k}}
$$

Here $\rho_{g}$ is the gas-phase density, $u_{j}$ the velocity vector, $p$ the pressure, $\mu$ the dynamic viscosity, $\delta_{i j}$ the Kronecker symbol, $Z$ the mixture fraction, $C$ the progress variable, $\alpha_{Z}$ and $\alpha_{C}$ the scalar diffusivities, and $\dot{\omega}_{C}$ the source term due to chemical reactions. The additional term 
in the continuity, $\overline{\dot{S}_{m}}$, mixture fraction, $\overline{\dot{S}}_{Z}$, and momentum equations, $\overline{\dot{S}}_{i}$, are the interphase mass and momentum transport terms. The unclosed transport terms in the momentum and scalar equations are grouped into the residual stress, $q_{i j}$, and residual scalar fluxes, $q_{Z j}, q_{C j}$. The filtering operation is denoted by an overbar and Favre (density-weighted) filtering by tilde. The choice of the progress variable depends on the flow conditions and chemistry. Typically, mass fractions of major product species is a good indicator of the forward 'progress' of the reaction.

\subsection{Presumed $P D F$ approach}

Following the FPV appraoch [5], the chemistry is incorporated in the form of a steady-state one-dimensional flamelet model. Due to the presence of the liquid phase, the transport equation for the mixture fraction (defined based on the fuel vapor) has a source term (Eq. 2). In addition, the heat of droplet vaporization is taken from the gas-phase causing evaporative cooling of the surrounding gas. This gives rise to a sink term in the energy equation. The evaporative cooling effect is accounted for during the generation of the flamelet tables by computing an effective gaseous fuel temperature, $T_{f u e l, g}=T_{f u e l, l}-L_{v a p} / C_{p l}$, where subscript $l$ stands for liquid, $L_{v a p}$ is the latent heat of vaporization, and $C_{p l}$ the specific heat of liquid fuel, and $T_{f u e l, l}$ the inlet liquid fuel temperature. This effective gaseous fuel temperature is used as boundary condition in solving the flamelet equations. By assuming adiabatic walls and unity Lewis number, the energy and mixture fraction equations have the same boundary conditions and are linearly dependent. The energy conservation equation is not solved in this formulation.

The subgrid fluctuations in the mixture fraction and progress variable, filtered combustion variables are obtained by integrating chemical state relationships over the joint $P D F$ of $Z$ and $C$. As an example, the filtered chemical source term of the progress variable is given as,

$$
\overline{\dot{\omega}}_{C}=\int \dot{\omega}_{C}(Z, C) \tilde{P}(Z, C) d Z d C
$$

The joint subgrid $P D F$ is modeled by writing, $\tilde{P}(Z, C)=\tilde{P}(C \mid Z) \tilde{P}(Z)$. Here, $\tilde{P}(Z)$ is modeled 
by the presumed beta subgrid $P D F$ and the conditional $P D F, \tilde{P}(C \mid Z)$ is modeled as a delta function according to Pierce \& Moin [5]. In the present two-phase flow application, the mixture fraction equation (Eq. 2) consists of a source term due to the evaporation of liquid fuel. By assuming a beta $P D F$ for $\tilde{P}(Z)$ we implicitly assume that the time-scale of evaporation is small compared to the scalar mixing time-scale. More advanced micro-mixing models accounting for spray-chemistry interactions are necessary [12] to better represent the filtered source terms in the continuity and mixture-fraction equations. Following these assumptions, the flamelet library is computed and subgrid $P D F$ integrals are evaluated to generate lookup tables to provide filtered variables as:

$$
\tilde{y}_{i}=\tilde{y}_{i}\left(\tilde{Z}, \widetilde{Z^{\prime \prime 2}}, \tilde{C}\right), \quad \tilde{T}=\tilde{T}\left(\tilde{Z}, \widetilde{Z^{\prime \prime 2}}, \tilde{C}\right), \quad \bar{\rho}_{g}=\bar{\rho}_{g}\left(\tilde{Z}, \widetilde{Z^{\prime \prime 2}}, \tilde{C}\right), \quad \text { etc. }
$$

where $\widetilde{Z^{\prime \prime 2}}$ is the mixture fraction variance, $\tilde{y}_{i}$ the species mass fractions, and $\tilde{T}$ the temperature. Similar expressions are obtained for dynamic viscosity, molecular diffusivities, and other properties required in the computation.

\subsection{Subgrid Scale Models}

The dynamic Smagorinsky model by Moin et al. [13] is used to close the subgrid terms as demonstrated by Pierce \& Moin [14]. The unclosed terms in Eqs. (2-4) are modeled using the eddy-viscosity assumption. The eddy viscosity, eddy diffusivities, and subfilter variance of the mixture fraction are evaluated as:

$$
\mu_{t}=C_{\mu} \bar{\rho}_{g} \Delta^{2}|\tilde{\mathbf{S}}|, \quad \bar{\rho}_{g} \alpha_{t}=C_{\alpha} \bar{\rho}_{g} \Delta^{2}|\tilde{\mathbf{S}}|, \quad \bar{\rho}_{g} \widetilde{Z^{\prime \prime 2}}=C_{\tilde{Z}} \bar{\rho}_{g} \Delta^{2}|\nabla \tilde{Z}|^{2}
$$

where $|\tilde{\mathbf{S}}|=\sqrt{\tilde{S}_{i j} \tilde{S}_{i j}}$. The coefficients $C_{\mu}, C_{\alpha}$, and $C_{\tilde{Z}}$ are evaluated dynamically [14]. 


\subsection{Liquid-Phase Equations}

The droplet motion is simulated using the Basset-Boussinesq-Oseen (BBO) equations [15]. It is assumed that the density of the droplet is much larger than that of the fluid $\left(\rho_{p} / \rho_{g} \sim 10^{3}\right)$, droplet-size is small compared to the turbulence integral length scale, and that the effect of shear on droplet motion is negligible. The high value of density ratio implies that the Basset force and the added mass term are small and are therefore neglected. Under these assumptions, the Lagrangian equations governing the droplet motions become

$$
\begin{gathered}
\frac{d \mathbf{x}_{p}}{d t}=\mathbf{u}_{p}, \\
\frac{d \mathbf{u}_{p}}{d t}=D_{p_{\text {drop }}}\left(\mathbf{u}-\mathbf{u}_{p}\right)+\left(1-\frac{\rho_{g}}{\rho_{p}}\right) \mathbf{g}
\end{gathered}
$$

where $\mathbf{x}_{p}$ is the position of the droplet centroid, $\mathbf{u}_{p}$ the droplet velocity components, $\mathbf{u}$ the gasphase velocities interpolated to the droplet location, $\rho_{p} \& \rho_{g}$ the droplet and gas-phase densities, and $\mathbf{g}$ the gravitational acceleration. The drag force on a droplet is modeled by drag coefficient, $C_{d}$, based on a solid particle with modifications due to internal circulation and deformation,

$$
D_{p_{\text {solid }}}=\frac{3}{4} C_{d} \frac{\rho_{g}}{\rho_{p}} \frac{\left|\mathbf{u}_{g}-\mathbf{u}_{p}\right|}{d_{p}}
$$

where $C_{d}$ is obtained from the nonlinear correlation [15]

$$
C_{d}=\frac{24}{R e}\left(1+a R e_{p}^{b}\right)
$$

Here $R e_{p}=d_{p}\left|\mathbf{u}_{g}-\mathbf{u}_{p}\right| / \mu_{g}$ is the particle Reynolds number. The above correlation is valid for $R e_{p} \leq 800$. The constants $a=0.15, b=0.687$ yield the drag within $5 \%$ from the standard drag curve. The above expression for solid body drag is modified to account for droplet deformation and internal circulation as given below.

In LES of droplet-laden flows, the droplets are presumed to be subgrid, and the droplet-size is smaller than the filter-width used. The gas-phase velocity field required in Eq. (10) is the total 
(unfiltered) velocity, however, only the filtered velocity field is computed in Eqs. (4). The direct effect of unresolved velocity fluctuations on droplet trajectories depends on the droplet relaxation time-scale, and the subgrid kinetic energy. Pozorski et al. [16] performed a systematic study of the direct effect of subgrid scale velocity on particle motion for forced isotropic turbulence. It was shown that, in poorly resolved regions, where the subgrid kinetic energy is more than

$30 \%$, the effect on droplet motion is more pronounced. A stochastic model reconstructing the subgrid-scale velocity in a statistical sense was developed [16]. In the present work, we neglect this direct effect of subgrid scale velocity on the droplet motion. However, note that the particles do feel the subgrid scales through the subgrid model that affects the resolved velocity field. For swirling, separated flows with the subgrid scale energy content much smaller than the resolved scales, the direct effect was shown to be small [9].

\subsubsection{Deformation and drag models}

The drag law for spherical, solid objects (Eq. 11) needs modifications when applied to liquid droplets in a turbulent flow. Droplet deformation and internal circulation may affect the drag force significantly. In order to quantify the effect of droplet deformation on drag, Helenbrook \& Edwards [17] performed detailed resolved simulations of axisymmetric liquid drops in uniform gaseous stream. Based on their computations for a range of density and viscosity ratios, range of Weber ( We), Ohnesorge (Oh), and Reynolds numbers (Re), a correlation was developed that provides the amount of droplet deformation in the form of ellipticity, $E$, which is defined as the ratio of the height to width of the drop,

$$
E=1-0.11 W e^{0.82}+0.013 \sqrt{\frac{\rho_{p}}{\rho_{g}}} \frac{\mu_{g}}{\mu_{l}} O h^{-0.55} W e^{1.1}
$$

where $\mu_{l}, \mu_{g}$ are the viscosities, and $\rho_{p}, \rho_{g}$ the densities of the liquid and gas-phase, respectively. The non-dimensional Weber and Ohnesorge numbers are defined as, $W e=\rho_{g} U^{2} d_{p} / \sigma$ and $O h=\mu_{l} / \sqrt{\rho_{p} \sigma d_{p}}$, where $U$ is the relative velocity between the gas and liquid, $d_{p}$ the diameter of the droplet, and $\sigma$ the surface tension. Accordingly, $E<1$ indicates that the drops have 
more width than height with deformation in a direction perpendicular to the relative velocity. These shapes are called oblate shapes. Similarly, $E>1$ gives elongation in the direction of the relative velocity giving rise to prolate shapes. $E=1$ implies spherical shapes.

The effect of droplet deformation is reflected in the drag force. This effect is modeled by using an effective equatorial droplet diameter, $d_{p}^{*}=d_{p} E^{-1 / 3}$. The particle Reynolds number is also modified, $R e_{p}^{*}=R e_{p} E^{-1 / 3}$. This is used in Eqs. $(11,12)$ to obtain the modified drag [17]. In addition the effect of internal circulation is modeled by changing the drag on a solid sphere as

$$
\frac{D_{p_{\text {drop }}}}{D_{p_{\text {solid }}}}=\left(\frac{2+3 \mu_{l} / \mu_{g}}{3+3 \mu_{l} / \mu_{g}}\right)\left(1-0.03\left(\mu_{g} / \mu_{l}\right) R e_{p}^{0.65}\right)
$$

\subsubsection{Stochastic model for secondary breakup}

Performing simulations of primary atomization where one tracks the liquid-gas interface in realistic combustor geometries is computationally intensive. The current state-of-the art is to compute the atomization process using subgrid, secondary breakup models based on point-particle approximation. Emphasis is placed on obtaining the correct spray evolution characteristics such as liquid mass flux, spray angle, and droplet size distribution. The liquid jet/sheet is approximated by large drops with size equal to the nozzle diameter. The effect of high mass-loading on the gas-phase momentum transport is captured through two-way coupling between the two phases.

A stochastic spray breakup model capable of generating a broad range of droplet sizes at high Weber numbers has been developed [10]. In this model, the characteristic radius of droplets is assumed to be a time-dependent stochastic variable with a given initial size-distribution. The breakup of parent drops into secondary droplets is viewed as the temporal and spatial evolution

of this distribution function around the parent-droplet size according to the Fokker-Planck (FP) differential equation:

$$
\frac{\partial T(x, t)}{\partial t}+\nu(\xi) \frac{\partial T(x, t)}{\partial x}=\frac{1}{2} \nu\left(\xi^{2}\right) \frac{\partial^{2} T(x, t)}{\partial x^{2}} .
$$


where the breakup frequency $(\nu)$ and time $(t)$ are introduced. Here, $T(x, t)$ is the distribution function for $x=\log \left(r_{p}\right)$, and $r_{p}$ is the droplet radius. Breakup occurs when $t>t_{\text {breakup }}=1 / \nu$. This distribution function follows a certain long-time behavior, which is characterized by the dominant mechanism of breakup. The value of the breakup frequency and the critical radius of breakup are obtained by the balance between the aerodynamic and surface tension forces. The secondary droplets are sampled from the analytical solution of Eq. (15) corresponding to the breakup time-scale. The parameters encountered in the FP equation $\left(\langle\xi\rangle\right.$ and $\left.\left\langle\xi^{2}\right\rangle\right)$ are computed by relating them to the local Weber number for the parent drop, thereby accounting for the capillary forces and turbulent properties [10]. As new droplets are formed, parent droplets are destroyed and Lagrangian tracking in the physical space is continued till further breakup events.

\subsubsection{Evaporation model}

Typical spray simulations do not resolve the temperature and species gradients around each droplet to compute the rate of evaporation. Instead, evaporation rates are estimated based on quasi-steady analysis of a single isolated drop in a quiescent environment $[18,19]$. Multiplicative factors are then applied to consider the convective and internal circulation effects. We model the droplet evaporation based on a 'uniform-state' model. The Lagrangian equations governing particle mass and heat transfer processes are well summarized by Oefelein [20] and are described here in brief.

$$
\begin{gathered}
\frac{d}{d t}\left(m_{p}\right)=-\dot{m}_{p} \\
m_{p} C_{p_{l}} \frac{d}{d t}\left(T_{p}\right)=h_{p} \pi d_{p}^{2}\left(T_{g}-T_{p}\right)-\dot{m}_{p} \Delta h_{v}
\end{gathered}
$$

where $\Delta h_{v}$ is the latent heat of vaporization, $m_{p}$ mass of the droplet, $T_{p}$ temperature of the droplet, and $C_{p_{l}}$ the specific heat of liquid. The diameter of the droplet is obtained from its 
mass, $d_{p}=\left(6 m_{p} / \pi \rho_{p}\right)^{1 / 3}$. The effective heat-transfer coefficient, $h_{p}$ is defined as,

$$
h_{p}=k_{s}\left(\frac{d T}{d r}\right)_{s g} /\left(T_{g}-T_{s}\right)
$$

where $k_{s}$ is the effective conductivity of the surrounding gas at the droplet surface. The subscript ' $s$ ' stands for the surface of the droplet. The expression for $\dot{m}_{p}$ and solution to Eqs. $(16,17)$ in quiescent medium are obtained by defining Spalding mass and heat transfer numbers and making use of the Clausius-Clapeyron's equilibrium vapor-pressure relationship [18]. In addition, convective correction factors are applied to obtain spray evaporation rates at high Reynolds numbers [20].

\subsubsection{Hybrid particle-parcel technique for spray simulations}

Performing spray breakup computations using Lagrangian tracking of each individual droplet gives rise to a large number of droplets $(\approx 20-50$ million $)$ in localized regions very close to the injector. Simulating all droplet trajectories gives severe load-imbalance due to presence of droplets on only a few processors. On the other hand, correct representation of the fuel vapor distribution obtained from droplet evaporation is necessary to capture the dynamics of spray flames. We have developed a hybrid particle-parcel scheme to effectively reduce the number of particles tracked and yet represent the overall spray evolution properly [10].

A parcel or computational particle represents a group of droplets, $N_{\text {par }}$, with similar characteristics (diameter, velocity, temperature). The basic idea behind the hybrid-approach is as follows. At every time step, droplets of the size of the spray nozzle are injected based on the fuel mass flow rate. New particles added to the computational domain are pure drops $\left(N_{\text {par }}=1\right)$. These drops are tracked by Lagrangian particle tracking and undergo breakup according to the stochastic model creating new droplets of smaller size. As the local droplet number density exceeds a prescribed threshold, all droplets in that control volume are collected and grouped into bins corresponding to their size and other properties such as velocity, temperature etc. The droplets in bins are then used to form a parcel by conserving mass, momentum and energy. The 
properties of the parcel are obtained by mass-weighted averaging from individual droplets in the bin. The number of parcels created would depend on the number of bins and the threshold value used to sample them. A parcel thus created then undergoes breakup according to the above stochastic sub-grid model, however, does not create new parcels. On the other hand, $N_{\text {par }}$ is increased and the diameter is decreased by mass-conservation.

This strategy effectively reduces the total number of computational particles in the domain. Regions of low number densities are captured by individual droplet trajectories, giving a more accurate spray representation.

\subsection{Interphase Exchange Terms}

The source terms in the gas-phase continuity, mixture-fraction, and momentum equations are obtained from the equations governing droplet dynamics (Eqs. 10,16). For each droplet the source terms are interpolated from the particle position $\left(\mathbf{x}_{\mathbf{p}}\right)$ to the centroid of the grid control volume using an interpolation operator. The source terms in the continuity and mixture fraction equations are identical as they represent conservation of mass of fuel vapor. The expressions for source terms are:

$$
\begin{array}{r}
\bar{S}_{m}(\mathbf{x})=\overline{\dot{S}}_{Z}(\mathbf{x})=-\frac{1}{\mathrm{~V}_{\mathrm{cv}}} \sum_{k} \mathcal{G}_{\sigma}\left(\mathbf{x}, \mathbf{x}_{\mathbf{p}}\right) \frac{d}{d t}\left(m_{p}\right) \\
\overline{\dot{S}}_{i}(\mathbf{x})=-\frac{1}{\mathrm{~V}_{\mathrm{cv}}} \sum_{k} \mathcal{G}_{\sigma}\left(\mathbf{x}, \mathbf{x}_{\mathbf{p}}\right) \frac{d}{d t}\left(m_{p} u_{p_{i}}^{k}\right)
\end{array}
$$

where the summation is over all droplets $(k), \mathrm{V}_{\mathrm{cv}}$ is the volume of the grid cell in which the droplet lies, and the function $\mathcal{G}_{\sigma}$ is the interpolation operator given as

$$
\mathcal{G}_{\sigma}\left(\mathbf{x}, \mathbf{x}_{p}\right)=\frac{1}{(\sigma \sqrt{2 \pi})^{3}} \exp \left[-\frac{\sum_{i=1}^{3}\left(x_{i}-x_{p_{i}}\right)^{2}}{2 \sigma^{2}}\right]
$$

where $\sigma$ is proportional to the grid size in which the droplet lies. For each droplet, the conser-

vation constraint, $\int_{\mathrm{V}_{\mathrm{cv}}} \mathcal{G}_{\sigma}\left(\mathbf{x}, \mathbf{x}_{p}\right) d V=1$, is imposed by normalizing the interpolation operator. 


\section{Numerical Method}

A co-located, finite-volume, energy-conserving numerical scheme on unstructured grids has been developed by Mahesh et al. [1] to solve the gas-phase incompressible flow equations. The velocity and pressure are stored at the centroids of the control volumes. The cell-centered velocities are advanced in a predictor step such that the kinetic energy is conserved. The predicted velocities are interpolated to the faces and then projected. Projection yields the pressure potential at the cell-centers, and its gradient is used to correct the cell and face-normal velocities. A novel discretization scheme for the pressure gradient was developed by Mahesh et al. [1] to provide robustness without numerical dissipation on grids with rapidly varying elements. This algorithm was found to be imperative to perform LES at high Reynolds numbers in realistic combustor geometries.

This formulation has been shown to provide very good results for both simple and complex geometries [1] and is extended to variable density, low Mach number equations to compute turbulent reacting flows [11]. In addition, for two-phase flows the particle centroids are tracked using the Lagrangian framework. The particle equations are integrated using third-order Runge-Kutta schemes. After obtaining the new particle positions, the particles are relocated, particles that cross interprocessor boundaries are duly transferred, boundary conditions on particles crossing boundaries are applied, source terms in the gas-phase equation are computed, and the computation is further advanced. Solving these Lagrangian equations thus requires addressing the following key issues: (i) efficient search for locations of particles on an unstructured grid, (ii) interpolation of gas-phase properties to the particle location for arbitrarily shaped control volumes, (iii) inter-processor particle transfer. An efficient Lagrangian framework was developed which allows tracking millions of particle trajectories on unstructured grids [9].

\section{Results: Validation Studies}

A systematic approach is taken to perform validation studies to assess the predictive capa-

bility of the numerical scheme used as well as different physical models described in section 2 . 
The basic idea is to isolate physical phenomena of droplet/particle motion, droplet breakup, evaporation, and turbulent combustion and simulate them in canonical problems where detailed description of the boundary conditions and in-depth experimental data are available. This also allows development and testing of new models and numerical schemes for flows of interest. The following cases with well specified boundary conditions have been simulated: 1) swirling, particle-laden flow in a co-annular jet (experiments by Sommerfeld \& Qiu [21]), 2) evaporating droplets in a turbulent flow (experiments by Sommerfeld \& Qiu [22]), 3) high-speed liquid jet atomization in a cylindrical chamber (experiments of Hiroyasu \& Kudota [23]), and 4) turbulent reacting flow with gaseous fuel (experiments by Spadaccini et. al. [24]). After verifying the predictive capability of the numerical scheme and models used for these flows, the following simulations in realistic combustor geometries were performed: 5) non-reacting, gaseous flow in a PW test rig representative of realistic conditions, 6) non-reacting flows in a real PW combustor, 7) liquid-spray patternation experiments of the PW injector, and 8) a multiphase, multiphysics simulation of reacting flow in real PW combustor.

In this work, emphasis is placed on validation of spray dynamics and details of cases 1-3, and 7-8 are presented next. The numerical results of case 4 and cases 5-6 have been well documented by Mahesh et al. $[1,11]$, respectively and are not repeated here.

\subsection{Validation of Eulerian-Lagrangian Formulation}

The Eulerian-Lagrangian formulation on unstructured grids is validated by simulating a swirling, particle-laden cold flow in a coaxial geometry corresponding to the experiments of Sommerfeld \& Qiu [21]. This flow configuration was chosen because the basic flow features with swirling jets and recirculation bubbles resemble those inside a gas-turbine combustor. In addition, the boundary conditions are very well specified along with detailed statistics for the gas and particle phase at various locations making it an important validation case.

Figure (1) shows a slice through the axisymmetric flow configuration which consists of a

central core (primary) and annular (secondary) jets discharging into a cylindrical test section 
with sudden expansion. The primary jet has a radius of $16 \mathrm{~mm}$ and is laden with glass beads with a mean number diameter $\left(D_{10}\right)$ of $45 \mu \mathrm{m}$ distributed between 10 and $120 \mu \mathrm{m}$ obtained by matching an upper-limit log-normal distribution with the experimental measurements. The secondary annular jet has a swirling azimuthal velocity and extends over the radial interval of 19-32 $\mathrm{mm}$. The outer radius of the annulus $(R)$ is $32 \mathrm{~mm}$, the whole test-section is $960 \mathrm{~mm}$ long, is oriented vertically with gravity acting in the direction of the flow and is connected to a stagnation chamber of length $640 \mathrm{~mm}$ and radius $288 \mathrm{~mm}$. The computational domain is divided in $\sim 1.6 \times 10^{6}$ hexahedral volumes and approximately 96 grid points are used in the azimuthal direction. The Reynolds number of the primary jet is 26200 , the particle loading ratio of 0.034 is too small to have dense clusters of particles, and the particle-particle interactions are neglected.

Figure (1) also shows the instantaneous contours of normalized axial velocity in the symmetry plane $(z=0)$ along with particle locations superimposed on the turbulent velocity field. The size of the circles scales with the actual diameter of the particles. The velocity field is normalized by the average inlet velocity of $12.82 \mathrm{~m} / \mathrm{s}$. The expansion of the swirling jet, central and secondary recirculation regions are clearly visible. The gas-phase inflow conditions are generated by simulating a periodic pipe and annulus with the desired mass flowrates and Reynolds numbers. Each particle injected into the computational domain is simulated giving around 1.1 million particles at stationary state. Close to the entrance of the test-section at $x / R=0$, particles are concentrated in the central core region. Particles are injected with the same velocities as the gas-phase at the inlet, however, majority of them do not follow the rapid expansion and deceleration of the gas jets. These particles are convected downstream and spread in the radial and azimuthal directions, some of them are trapped in the central recirculation region. Particles tend to have higher velocities in the core region, penetrate the central recirculation region, move against the gas-phase flow till they are decelerated to zero axial velocity. These particles then change their directions, and are thrown radially outwards by the centrifugal action. Heavier particles penetrate the recirculation bubble the most because of their larger inertia. The basic features of particle-laden, swirling flows, such as the recirculation bubble length, the 
reattachment points, the trajectories of different particle sizes are accurately captured [9].

It is important to note that accurate prediction of these flow features plays an important role in investigating swirling, turbulent flows in gas-turbine combustors. Figure (2) shows comparison with the experimental data of azimuthally averaged particle-phase mean and $\mathrm{rms}$ axial, swirl, and radial velocity at different cross-sections. Also shown are the mean and $r m s$ particle diameter distributions indicating good agreement. Important particle-dispersion characteristics are obtained from this simulation to further investigate swirling, particle-laden flows [9]. It is also confirmed that LES together with the point-particle approach for dispersed phase can accurately predict swirling, separated flows for dilute-particle loadings.

\subsection{Validation of evaporation model and variable density formulation}

In order to validate the evaporation model and the variable density formulation, simulation of a coaxial non-swirling jet has been performed following the configuration used in the experiments of Sommerfeld \& Qiu [22]. This experiment also is well documented with specified boundary conditions for both phases. For the liquid phase the inlet droplet size distribution and their correlation with droplet velocity are well-defined. The gas-phase temperatures are not high enough to produce spray flames. This isolates the droplet evaporation problem from spray breakup and combustion, and makes it suitable to validate the evaporation models.

Figure (3) shows a snapshot of fuel mass fraction contours superimposed by scatter plot of Isopropyl alcohol droplets in the symmetry plane $(z=0)$. Hot air at $373 \mathrm{~K}$ is injected through the annulus and liquid droplets at $313 \mathrm{~K}$ are injected along the central wall region according to the measured size-velocity correlations at $x=3 \mathrm{~mm}$ downstream of the wall [22]. The grid used consists of $1.5 \mathrm{M}$ hexahedral cells and around $0.75 \mathrm{M}$ particles are present in the computational domain at statistically stationary state. The droplets are injected near the inlet circular wall of cross-sectional radius $R=20 \mathrm{~mm}$. The droplet velocity-size correlation depicts a conical spray with a spray angle of around $60^{\circ}$. The Reynolds number at inlet annulus is 21164 and the gas-phase inflow velocity is obtained by pre-computing a periodic annulus flow at same Reynolds number and mass-flow rate and storing instantaneous velocity profiles over 10 flow 
through times. A convective boundary condition is used at the exit plane. The combustor walls exhibit some heat losses as measured by the experiments, however, these are assumed small and adiabatic conditions are applied.

Figure (4) shows the radial variations of the mean and $r m s$ droplet velocity, and the mean and $r m s$ of droplet diameter compared to the experimental data. At $x / R=0$ and $x / R=0.786$ the profiles of the mean droplet axial velocity show two peaks associated to the hollow-cone spray. The droplet mean diameter shows a typical result corresponding to a hollow cone atomizer, where smaller droplets are found in the core region and larger droplets near the edge of the spray. Away from the injector, the spreading of the spray is hindered by the annular air-jets, the droplet mean diameter becomes more uniform over the cross-section and slowly decreases in the downstream direction because of evaporation. The axial mass-flux (not shown) also decreases toward the exit due to evaporation and is well captured by the present simulation.

To further validated the the low-Mach number, variable density formulation on unstructured grids at higher density ratios, a non-premixed methane-air flame in a coaxial combustor has been simulated. Details of this simulation have been addressed by Mahesh et al. [11] and are not presented here.

\subsection{Validation of stochastic breakup model}

To validate the stochastic breakup model together with the hybrid algorithm, a standard test case for high-speed liquid atomization in a cylindrical chamber is simulated. The computational domain is a closed cylindrical chamber of length $13.8 \mathrm{~cm}$ and diameter $5.6 \mathrm{~cm}$, following the experimental setup by Hiroyasu and Kudota [23]. Liquid jet is injected through a single-hole nozzle into this constant pressure, room-temperature nitrogen chamber. Since the chamber temperature is low, evaporation of the liquid fuel is negligible. The initial droplet diameter is $300 \mu \mathrm{m}$ corresponding to the nozzle size. The number of droplets injected per iteration is determined based on the droplet diameter and time-step by keeping the mass flow rate constant. The time step used in this simulation is $15 \mu$ s and a uniform grid of $100 \times 65 \times 65$ cells is found to capture the spray dynamics accurately. This configuration was chosen because of its simplicity 
and several different models have been calibrated against this experiment. In addition, the gasphase is initially at rest, and any flow generated is through the momentum transfer from the liquid to the gas-phase allowing us to investigate the two-way coupling effects. The gas-phase flow generated alters the relative velocity between the droplets and the flow, changes the breakup time-scale and plays an important role in capturing the spray penetration depth accurately.

The simulation was carried out at three different chamber pressures using the hybrid approach outlined in section 2.4.4. Figure (5) shows the time evolution of liquid spray at 1.1MPa. The region close to the injector mostly consists of large unbroken drops along with small, stripped droplets. The ligament-like liquid structures deflected outward are clearly visible. Comparison of penetration depth to experimental data at different chamber pressures obtained from the hybrid approach is shown in Fig. (6). Other spray characteristics such as liquid core length, spray angle, and the Sauter mean diameter (SMD) are also in good agreement with the experimental data $[10]$.

\subsection{Validation of spray patternation study for realistic PW injector}

The stochastic model along with the hybrid particle-parcel approach are used to compare the prediction of spray patternation characteristics for a realistic PW injector with the experimental data. The experimental data set [25] was obtained by mounting the actual injector in a cylindrical plenum through which gas with prescribed mass-flow rate was injected. Figure (7a) shows a cut through the symmetry plane $(z=0)$ of the computational domain along with the mesh and boundary conditions used. For this case, $3.2 \mathrm{M}$ grid points are used with high resolution near the injector. The domain decomposition is based on the optimal performance of the Eulerian gas-phase solver on 96 processors. Air from the inlet plenum goes through the central core, guide, and outer swirlers to create multiple swirling jets. All the passages through the swirler are computed. Also shown are the instantaneous axial velocity contours superimposed by the droplet locations (Fig. 7b). The swirling air jet from the core swirler enters the dump region and forms a recirculation zone. Jets from guide and outer swirlers interact with the core flow. A complex vortex break down phenomenon is observed and its accurate prediction is necessary to 
correctly represent the injector flow. Substantial amount of air is entrained in the radial direction and the rate of air entrainment is specified based on the experimentally measured values. Liquid film is injected through the filmer surface which forms an annular ring. In the symmetry plane this is indicated by two points on the edge of the annular ring (Fig. 7b). The liquid mass-flow rate corresponds to certain operating conditions of the gas-turbine engine. Actual numbers are not listed because of the proprietary nature of the experimental data. However, comparisons are presented in terms of normalized quantities or percentage errors.

The liquid film at the filmer surface, is approximated by injecting uniform size drops of the size of the annular ring thickness. These drops are convected downstream by the swirling air and undergo breakup according to the stochastic model. A large number of droplets are created in the vicinity of the injector due to breakup as shown in Fig. (7b). With the hybrid approach, the total number of computational particles tracked at stationary state is around $3.5 \mathrm{M}$ and includes around 150,000 parcels. Together these represent aproximately 13M droplets. Even with the hybrid approach, the load-imbalance due to atomization was found to be significant as only $1 / 3^{r d}$ of the processors had more than 10,000 computational particles. Dynamic loadbalancing techniques to reduce this computational overhead due to sprays are being investigated to improve the overall speedup [26].

Figure 8 compares LES predictions to the available experimental data of radial variations of mean axial and swirl velocity at different axial locations $(x=0.4, x=1.1$, and $x=2.1$, where $x$ is the distance from the injector wall normalized by the reference length scale). The numerical results are azimuthally averaged. The predictions from our simulation are in close agreement with the experimental data. Small disagreement at $x=2.1$ is partly related to the coarse grid resolution used away from the injector. It should be noted that the amount of swirl generator by the injectors determines the size of the recirculation zone. Good agreement of the axial and swirl velocities indicate that LES with dynamic sgs model can capture the vortex break-down phenomenon accurately in complex geometries. Also shown are the corresponding predictions using an in-house $k-\epsilon$ model. The unsteady RANS solutions are in agreement with the LES and experimental data very close to the injector, however, degrade rapidly further away, showing 
limitations of the turbulence model. Improved predictions using advanced RANS models can be obtained, however, the superiority of LES is clearly demonstrated. It should be noted that, any artificial dissipation or inaccurate numerics gives faster decay of the swirl velocities and incorrect size of the recirculation region, further emphasizing the importance of non-dissipative numerical schemes for LES.

Figure (9) compares radial variation of liquid mass-flowrates using LES and the stochastic model to the experimental data. The flow rates are presented as the ratio of the effective to the integrated flow rate. The effective flow rate is defined as the flow rate the patternator would record if the fuel flux was uniform at the local value. This normalization inherently carries the ratio of the total cross-sectional area to the area of the local patternator holes. Also shown are the predictions made by the $k-\epsilon$ model together with the Taylor-Analogy Breakup (TAB) model [27] commonly used in industry. The LES results are generally in good agreement with the experiments. Average droplet sizes at two axial location from the injector wall $(x=1.1$ and $x=2.1$ ) have been measured using the Malvern line of sight technique. The Sauter mean diameters averaged over the cross-section at these two axial locations are predicted within $5 \%$ of the experimental values. The present LES with stochastic breakup model predicts a broader distribution (not shown) as opposed to the one predicted by RANS with TAB model. The size distributions also indicate presence of large number of small size droplets compared to the experiments. This is attributed to the lack of collision/coalescence models in the present simulation. In addition, the initial droplet size at the injector nozzle is assumed to be a constant, whereas it may vary depending on the local conditions governing primary atomization. A further investigation with inclusion of collision models as well as using a size distribution at the inlet should be performed in order to investigate uncertainties in model predictions. 


\section{Validation of Multi-physics Reacting Flow Simulation in PW Combustor}

A multiscale, multiphysics simulation of turbulent reacting flow in a realistic Pratt \& Whitney combustor has been performed. This includes all the complex models for spray breakup, evaporation, and turbulent combustion described in section (2.4). Figure (10a) shows a section through the symmetry plane of the combustor geometry along with contours of temperature. Also shown are the instantaneous droplet locations in this plane forming a conical spray. The computational grid consists of $1.9 \mathrm{M}$ hybrid elements (hexes, pyramids, and tets) with fine resolution close to the injector. The grid inside the combustion chamber is hex-dominant to obtain good numerical accuracy. This simulation is performed on a single $\left(1 / 18^{\text {th }}\right)$ sector of the full combustor. The injector is same as the one used in the liquid fuel patternation study described earlier (section 4.4). The experimental data available for validation includes mass-splits through different components, temperature profile, and distribution of pollutants, NOx at the measurement plane.

Liquid fuel (Jet-A) enters the combustion chamber through an annular ring at the injector exit. This liquid film is approximated by large drops of the size of the injector annulus. These drops are convected by the surrounding hot air, they break, evaporate, and the fuel vapor thus formed mixes with the surrounding air giving a non-premixed spray flame. The flamelet library for Jet-A fuel at gas-turbine engine operating conditions, is generated by using a surrogate fuel (80\% n-Decane and 20\% 1-2-4 tri-methyl-benzene) chemistry which closely follows the chemical kinetics and reaction rates of the Jet-A fuel. Around 1000 elementary reactions among 100 chemical species are used to generate these tables. The chemical kinetics of surrogate fuel compared to the original fuel chemistry in terms of prediction of pollutants in laminar flames showed good agreement [28]. The progress variable for this simulation represents the summation of mass fractions of $\mathrm{CO}_{2}$ and $\mathrm{H}_{2} \mathrm{O}$.

Figure (10b) shows instantaneous snapshots of normalized temperature at various crosssections from the injector nozzle. High temperatures in the combustion chamber are reduced 
by cold air influx through the dilution holes. This reduces the exit temperature considerably. The prediction of mass-splits through different components was shown to be within $5 \%$ of the experimental data for the cold and reacting flow simulations $[1,11]$ and are not repeated here. In addition, the average temperature in the measurement plane is within $2 \%$ of the experimental data. The comparison of the radial variation of the mean temperature is shown in Fig. (11) and was obtained by taking azimuthal average over the sector. This represents our first attempt to perform such large-scale simulations in realistic configurations and the results give considerable insight into the combustor spray-flame dynamics.

This computation has been performed on 80 processors of ASC Frost (IBM SP3) and involved around $0.6 \mathrm{M}$ computational particles. It takes around 25-30 CPU-days on ASC Frost to obtain statistics over 40 time units (3-4 flow through times) starting from a statistically stationary solution. Significant advances in numerical algorithms resulting in efficient speedup of the solver are underway [26]. These new algorithms are expected to make possible such a multiphysics, multiphase computation of the full 18 injector combustor on $100 \mathrm{M}$ control volumes within 120 150 CPU-hrs on the full ASC Frost machine (1024 processors). In addition, advanced models for prediction of pollutants and NOx are being developed and applied to compute their distributions within the combustion chamber. A variety of flow conditions including cruise, high power, takeoff etc. are also being simulated for multiple sectors of the combustor.

\section{$6 \quad$ Summary \& Future Work}

A non-dissipative, energy-conserving numerical solver for arbitrary-shaped unstructured grids is developed to simulate multiphase, multiscale turbulent reacting flows in realistic gas-turbine combustors. This numerical tool solves low-Mach number, variable density flow equations for the gas-phase. Advanced models to capture atomization \& droplet breakup, droplet evaporation, droplet deformation \& drag, and turbulent combustion have been developed to simulate complex multiphase reacting flows. In this paper, we emphasize the necessity of performing detailed validation and verification studies to assess the predictive capability of the numerical 
solver as well as the models used. A series of validation cases in canonical configurations were selected to systematically evaluate each component of the numerical solver: particle-laden flows, evaporating droplets, gas-phase combustion, and droplet breakup. Results predicted show good agreement with the experimental data. Having verified the good predictive capability, a spray patternation study for PW injector and a multiphysics, multiscale simulation of reacting flow in one sector of the $\mathrm{PW}$ combustor were performed to give encouraging results and considerable insight into the unsteady flow development inside the combustor.

Advanced numerical schemes handling primary atomization and liquid sheet breakup [29], finite-size effects of the droplets in the dense-spray regime [30], models for liquid-film formation [31], droplet dispersion [16], models based on concepts of micro-mixing for droplet flame interactions, advanced combustion models for premixed/partially premixed turbulent flames, soot and pollutant formation, and radiation are being developed. In addition, a highly accurate numerical scheme capturing acoustic waves without the stringent acoustic CFL-restriction in compressible flows has been developed to investigate combustion instabilities [32]. An extensive effort is ongoing to make this tool efficient (by using multigrid solvers, dynamic load-balancing techniques), modular, user-friendly and capable of solving relevant industrial problems with quick turn around time to suit the demands of combustor design cycle in aircraft industry.

\section{Acknowledgement}

Support for this work was provided by the United States Department of Energy under the Accelerated Strategic Computing (ASC) program and NASA's UEET program. The computations were performed on Blue Horizon at San Diego supercomputing center and ASC Frost at Lawrence Livermore National Laboratory. We are grateful to the Pratt \& Whitney's combustor group for providing important data for validation. The help provided by Prof. Krishnan Mahesh

(University of Minnesota), Drs. Gianluca Iaccarino, Frank Ham, Prof. Heinz Pitsch (Stanford University), and Prof. George Constantinescu (University of Iowa) is highly appreciated. 


\section{References}

[1] Mahesh, K., Constantinescu, G., and Moin, P., "A New Time-Accurate FiniteVolume Fractional-Step Algorithm for Prediction of Turbulent Flows on Unstructured Hybrid Meshes," J. Comput. Phys., Vol. 197, 2004, pp. 215-240.

[2] Malecki, R.E., Rhie, C.M., Colket, M.B., Madabhushi, R.K., McKinney, R.G., Ouyang, H., And Syed, S.A., "Application of an Advanced CFD-Based Analysis System to the PW6000 Combustor to Optimize Exit Temperature Distribution Part I: Description and Validation of the Analysis Tool," ASME Turbo Expo, Paper No. 2001-GT-0062, 2001.

[3] Brankovic, A., McKinney, R.G., Ouyang, H., Porter, L., Kenndy, J., MadABhushi, R., And Colket, M., "Comparison of Measurements and Predictions of Flow in a Gas Turbine Engine Fuel Nozzle," AIAA Paper No. 2001-0331, 2000.

[4] Moin, P., and Mahesh, K., "Direct Numerical Simulation: a Tool in Turbulence Research," Ann. Rev. Fluid Mech., Vol. 30, 1998, pp. 539-578.

[5] Pierce, C.D., And Moin, P., "Progress-Variable Approach for Large-eddy Simulation of Turbulent Non-Premixed Combustion," J. Fluid Mech., Vol. 504, 2004, pp. 73-97.

[6] Kim, W.-W., And Syed, S., "Large-eddy Simulation Needs for Gas-Turbine Combustor Design," AIAA Paper No.2004-0331, AIAA Aerospace Sciences Meeting, Reno, NV, 2004.

[7] Mongia, H.. "Recent Advances in the Development of Combustor Design Tools," AIAA Paper No. 2003-4495.

[8] Mittal, R., and Moin, P., "Suitability of Upwind-Biased Finite Difference Schemes For Large-eddy Simulations of Turbulence Flows," AIAA J., Vol. 35, 1997, pp. 14151417. 
[9] Apte, S. V., Mahesh, K., Moin, P., and Oefelein, J.C. "Large-eddy Simulation of Swirling Particle-Laden Flows in a Coaxial-Jet Combustor," Int. J. Mult. Flow, Vol. 29, 2003, pp.1311-1331.

[10] Apte, S. V., Gorokhovski, M. And Moin, P., "LES of atomizing spray with stochastic modeling of secondary breakup," Int. J. Mult. Flow, Vol. 29, 2003, pp.1503-1522.

[11] Mahesh, K., Constantinescu, G., Apte, S.V., Iaccarino, G., Ham, F., and Moin, P., "Large-eddy Simulation of Reacting Turbulent Flows in Complex Geometries," ASME J. App. Mech., accepted for publication, 2005.

[12] Reveillon, J., And Vervisch, L. "Spray Vaporization in Non-premixed Turbulent Combustion Modeling: A Single Droplet Model," Comb. Flame, Vol. 121, 2000, pp.7590.

[13] Moin, P., Squires, K., Cabot, W., and Lee, S., "A dynamic subgrid model for compressible turbulence and scalar transport," Phy. Fluid A., Vol. 3, 1991, pp.27461757.

[14] Pierce, C.D., And Moin, P., "A Dynamic Model for Subgrid-Scale Variance and Dissipation Rate of a Conserved Scalar," Phy. Fluids, Vol.10, 1998, pp.3041-3044.

[15] Crowe, C., Sommerfeld, M., And Tsuji, Y., Multiphase Flows with Droplets and Particles, CRC Press, Boca Raton, FL, 1998.

[16] Pozorski, J., Apte, S.V., and Raman, V. "Filtered Particle Tracking for Dispersed Two-Phase Flows," Proceedings of the Summer Program, Center for Turbulence Research, Stanford University, 2004, (http://ctr.stanford.edu/).

[17] Helenbrook, B. T., And Edwards, C. F., "Quasi-steady Deformation and Drag of Uncontaminated Liquid Drops," Int. J. Multi. Flows., Vol. 28, 2002, pp.1631-1657.

[18] Faeth, G., "Current status of droplet and liquid combustion," Prog. Energy Combust. Sci., Vol. 3, 1977, pp.191-224.

[19] Faeth, G., "Evaporation and combustion of sprays," Prog. Energy Combust. Sci., Vol. 9, 1983, pp.1-76. 
[20] Oefelein, J. C., "Simulation and analysis of turbulent multiphase combustion processes at high pressures," Ph.D. Thesis, The Pennsylvania State University, University Park, Pa, 1997.

[21] Sommerfeld, M., AND QIu, H.H., "Detailed measurements in a swirling particulate two -phase flow by a phase - Doppler anemometer," Int. J. Heat E Fluid Flow, Vol. 12, No. 1, 1991, pp.20-28.

[22] Sommerfeld, M., And QIU, H.H., "Experimental studies of spray evaporation in turbulent flow," Int. J. Heat \& Fluid Flow, Vol. 19, 1998, pp.10-22.

[23] Hiroyasu, M., and Kudota, T., "Fuel droplet size distribution in diesel combustion chamber," SAE Tech. Paper: 740715, 1974.

[24] Spadaccini, L.J., Owen, F.K., and Bowman, C.T., "Influence of aerodynamic phenomena on pollutant formation in combustion (Phase I. Gaseous fuels)," U.S. Environmental Protection Agency Report EPA-600/2-76-247a, 1976.

[25] McKinney, R., Madabhushi, R.K., And Syed, S., private communication, 2002.

[26] Ham, F., Apte, S.V., Iaccarino, G., Wu, X., Herrmann, M., Constantinescu, G., Mahesh, K., And Moin, P., "Unstructured LES of Reacting Multiphase Flows in Realistic Gas-Turbine Combustors," Annual Research Briefs, Center for Turbulence Research, Stanford University, 2003, pp. 139-160, (http://ctr.stanford.edu).

[27] O'Rourke, P.J., And Amsden, A. A., " The TAB method for numerical calculations of spray droplet breakup," SAE Technical Paper, No. 87-2089, 1987.

[28] Riesmeier, E, Honnet S, Peters N "Flamelet Modeling of Pollutant Formation in a Gas Turbine Combustion Chamber Using Detailed Chemistry for a Kerosene Model Fuel," ASME J. Engr. Gas Turb. \& Power-Tran., Vol. 126, No. (4), 2004, pp.899-905.

[29] Herrmann, M., "A Level-Set/Vortex Sheet Method for Two-Phase Interface Dynamics," J. Comp. Phys., Available online, October, 2004. 
[30] Apte, S.V., Mahesh, K., And Lundgren, T., "A Eulerian-Lagrangian Model to Simulate Two-Phase Particulate Flows," Annual Research Briefs, Center for Turbulence Research, Stanford University, 2003, pp.161-171, (http://ctr.stanford.edu/).

[31] Kim, W.-W, Apte, S.V., Herrmann, M., and Ham, F. "Liquid Film Modeling in Jet Engine Fuel Injectors," Proceedings of the Summer Program, Center for Turbulence Research, Stanford University, 2004, (http://ctr.stanford.edu/).

[32] C. Wall, C. D. Pierce, And P. Moin, "A semi-implicit method for resolution of acoustic waves in low mach number flows". J. Comp. Phys., Vol. 181, No. (2), 2002, pp. $545-563$.

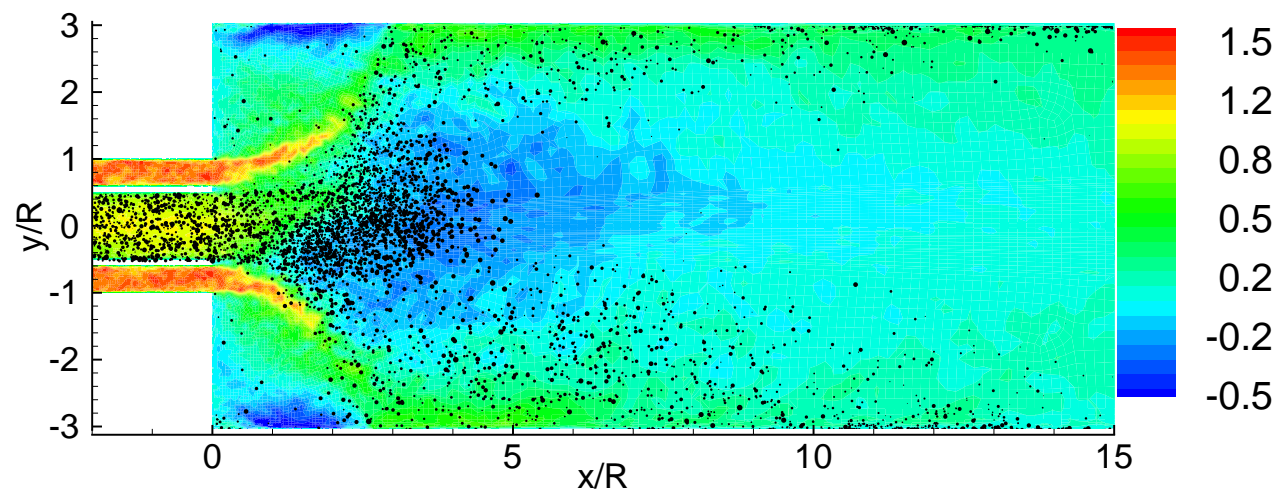

Figure 1: Snapshot of axial velocity contours superimposed by scatter plot of glass-particles in particle-laden, swirling flow in a co-axial jet combustor simulating experiment by Sommerfeld \& Qiu [21]. The velocity scale is normalized by average inlet velocity of $12.82 \mathrm{~m} / \mathrm{s}$. 


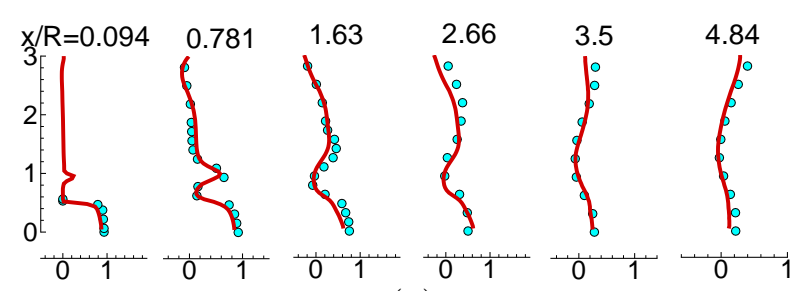

(a)

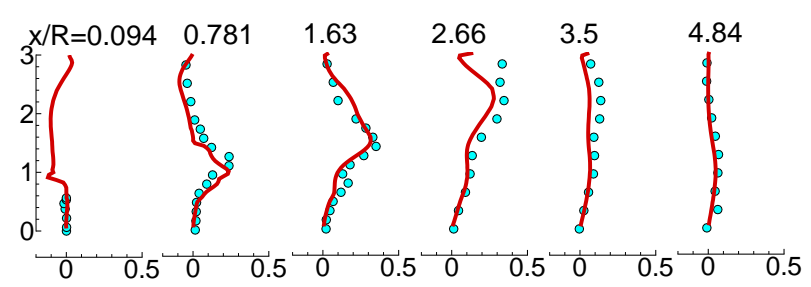

(c)

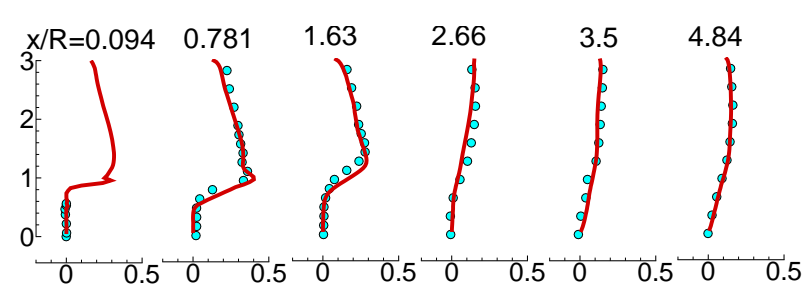

(e)

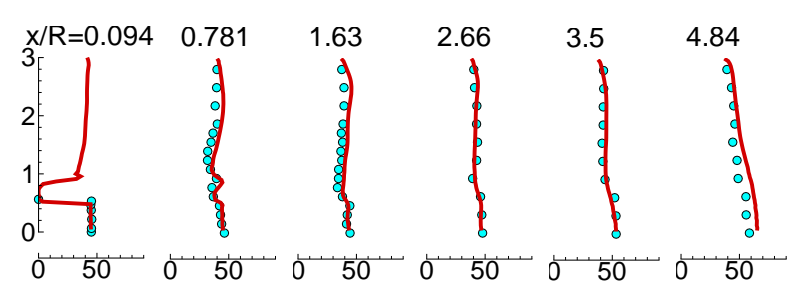

(g)

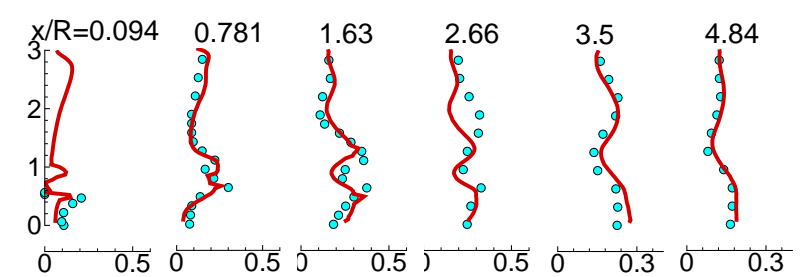

(b)

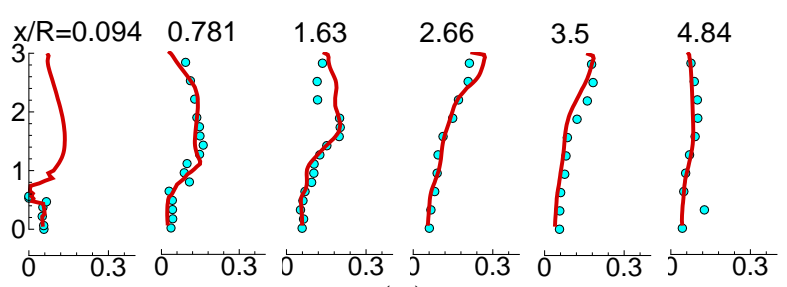

(d)

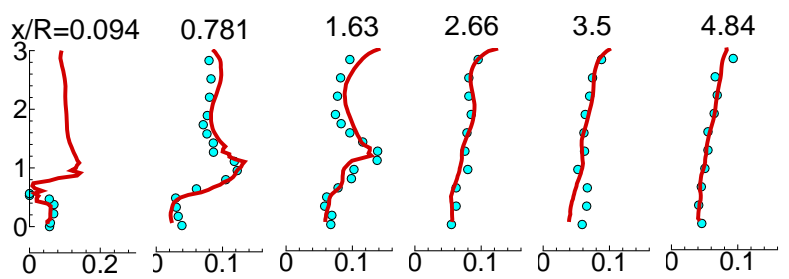

(f)

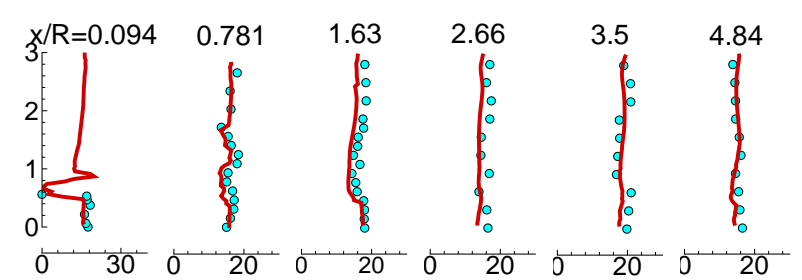

(h)

Figure 2: Comparison of particle statistics obtained from LES ( - ) to experimental data $(\circ)$ of Sommerfeld \& Qiu [21] at different axial locations ( $x$ normalized by the reference annulus radius $R=32 \mathrm{~mm}$ ): (a) mean axial velocity, (b) rms axial velocity, (c) mean radial velocity, $(d) \mathrm{rms}$ radial velocity, $(e)$ mean swirl velocity, $(f)$ rms of swirl velocity, $(g)$ mean diameter (microns), $(h) r m s$ of particle diameter (microns). 


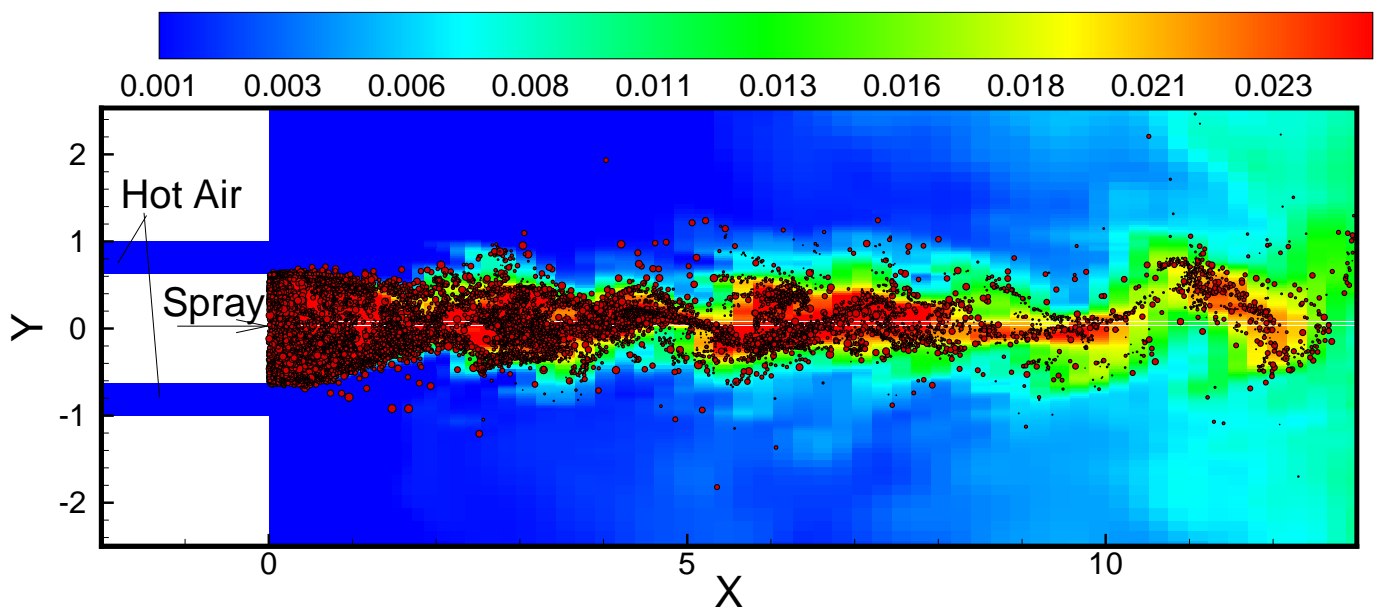

Figure 3: Snapshot of fuel mass fraction superimposed by droplet scatter plot in the symmetry plane $(z=0)$ of a co-axial combustor simulating experiment by Sommerfeld \& Qiu [22]. 

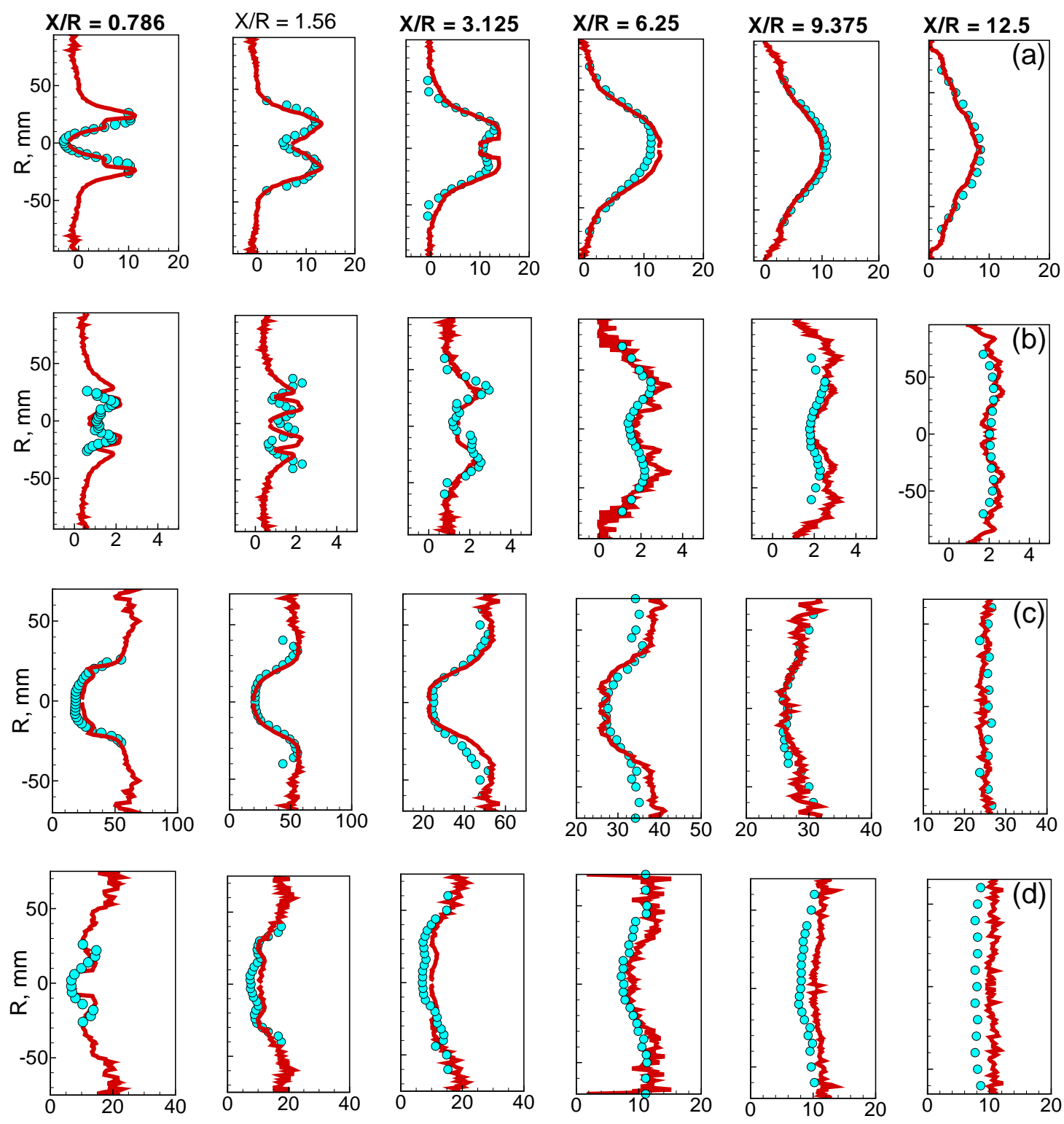

Figure 4: Radial variations of droplet statistics at different axial locations ( $x$ normalized by the core radius $R=20 \mathrm{~mm}$ ) compared to experimental data of Sommerfeld \& Qiu [22], LES, o experimental data: a) mean axial velocity $(\mathrm{m} / \mathrm{s})$ b) $r m s$ of axial velocity, $(\mathrm{m} / \mathrm{s}) \mathrm{c}$ ) mean diameter (microns), and d) rms diameter (microns). 

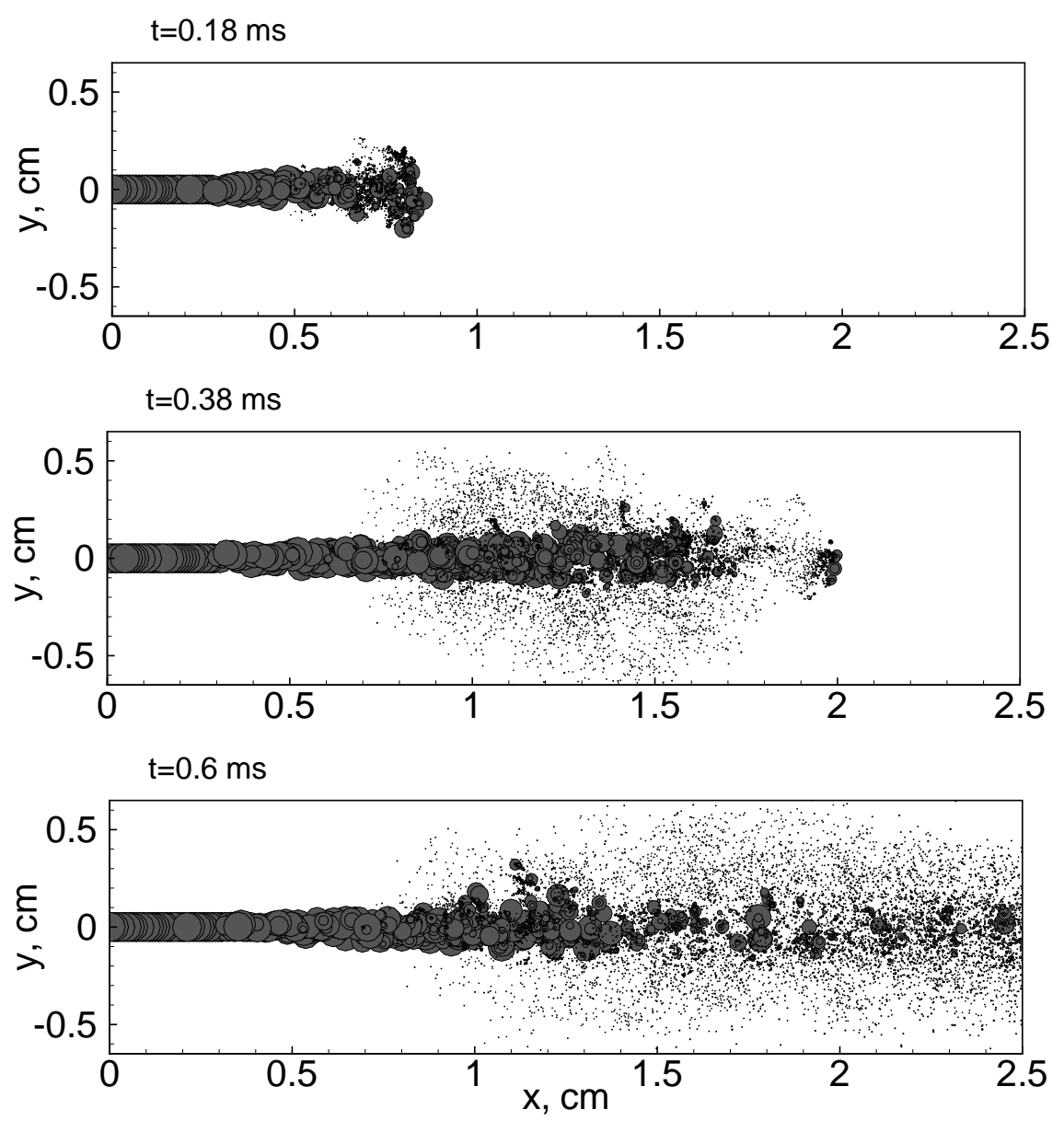

Figure 5: Time evolution of high speed liquid jet undergoing breakup in a closed cylindrical chamber at $\mathrm{P}=1.1 \mathrm{MPa}$. 


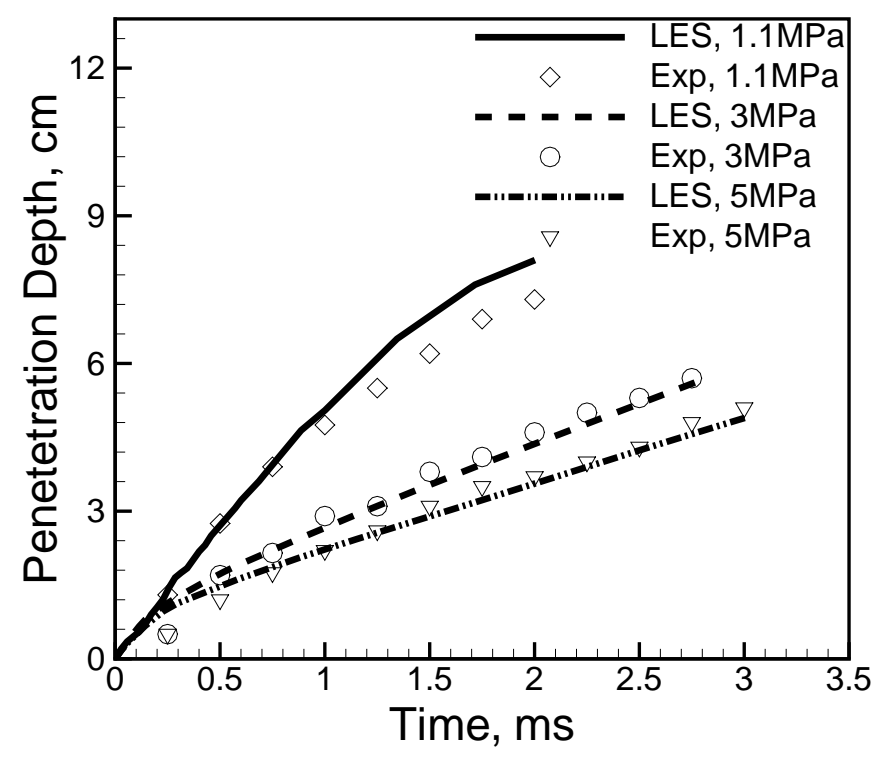

Figure 6: Validation of stochastic model with hybrid approach for atomization of high-speed liquid jet. Prediction of liquid spray penetration depth at different chamber pressures compared with experimental data by Hiroyasu \& Kudota [23]. 

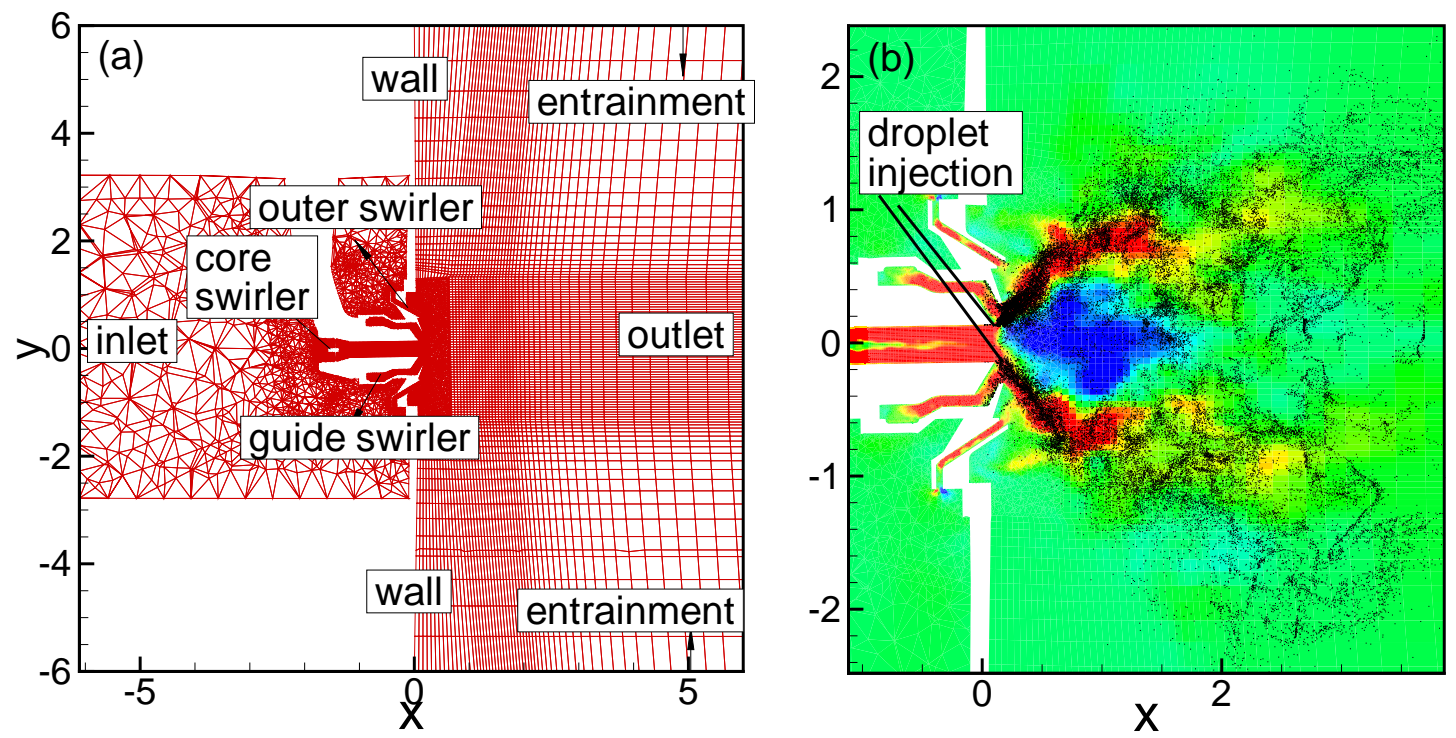

Figure 7: Snapshot of the symmetry plane $(z=0)$ of a PW injector: a) the full computational domain indicating boundary conditions and computational mesh, b) instantaneous droplet locations in the symmetry plane along with axial velocity contours. $x$ and $y$ are normalized axial and radial coordinates. 

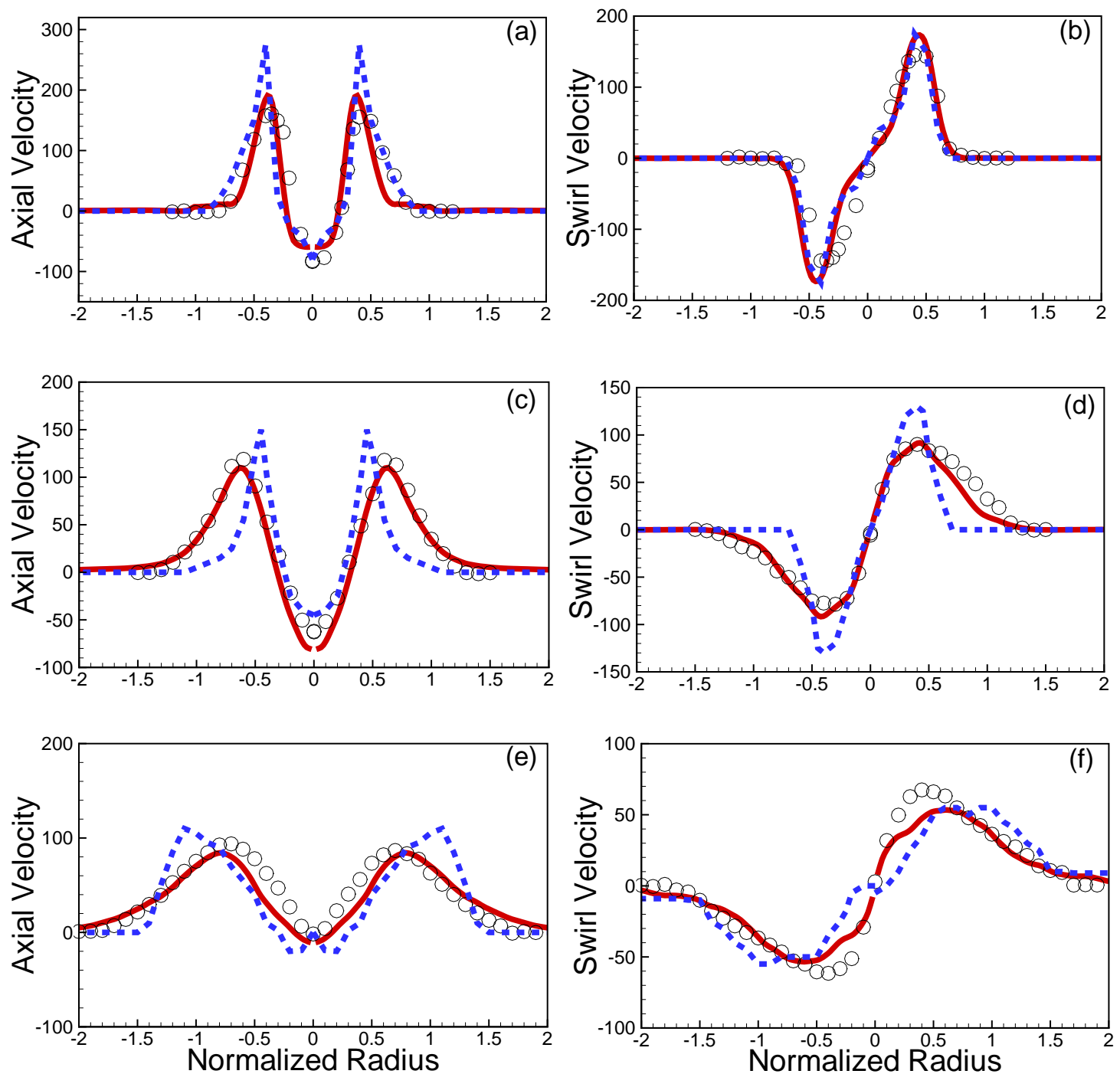

Figure 8: Radial variation of normalized mean axial and swirl velocities at different axial locations for the PW injector patternation study: o experimental data [25], LES, ---- Unsteady RANS using $k-\epsilon$ model: (a-b) $x=0.4$, (c-d) $x=1.1$, (e-f) $x=2.1$. Here $x$ is the normalized distance from the injector wall. 

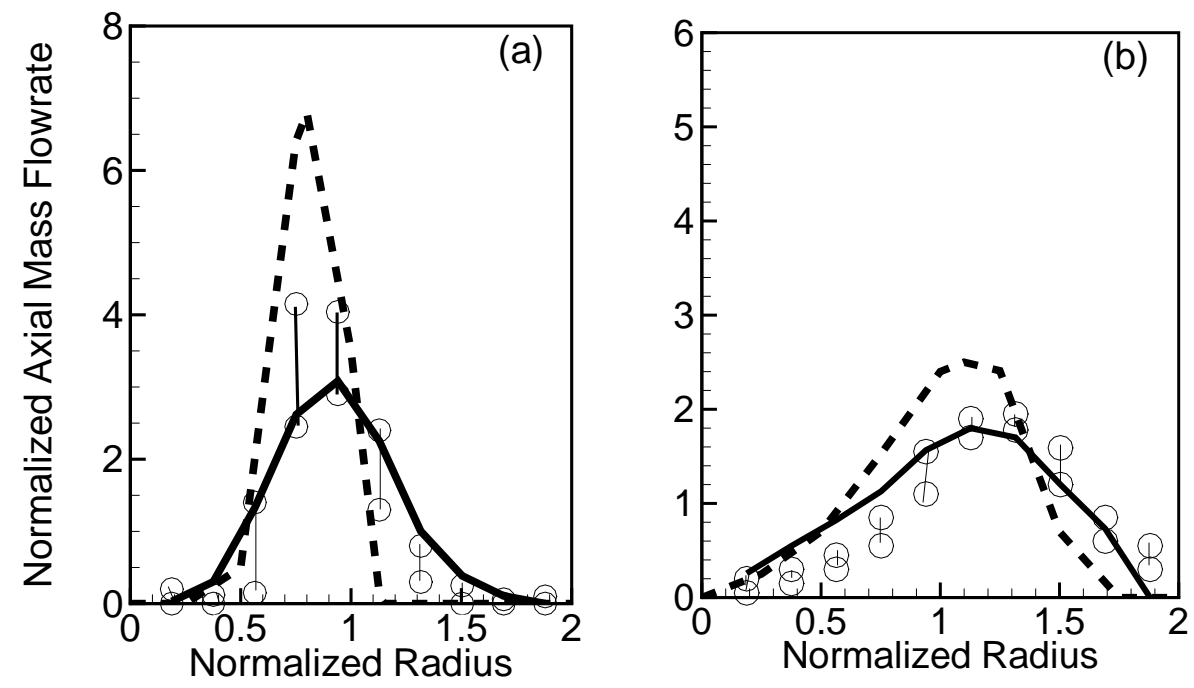

Figure 9: Comparison of radial variation of normalized liquid axial mass flux at two axial locations for the PW injector spray patternation study: ---- Unsteady RANS with TAB model for breakup, — present LES with stochastic breakup model, o — o experimental error bar: a) $x=1.1$, b) $x=2.1$ where $x$ is the normalized distance from the injector wall. 


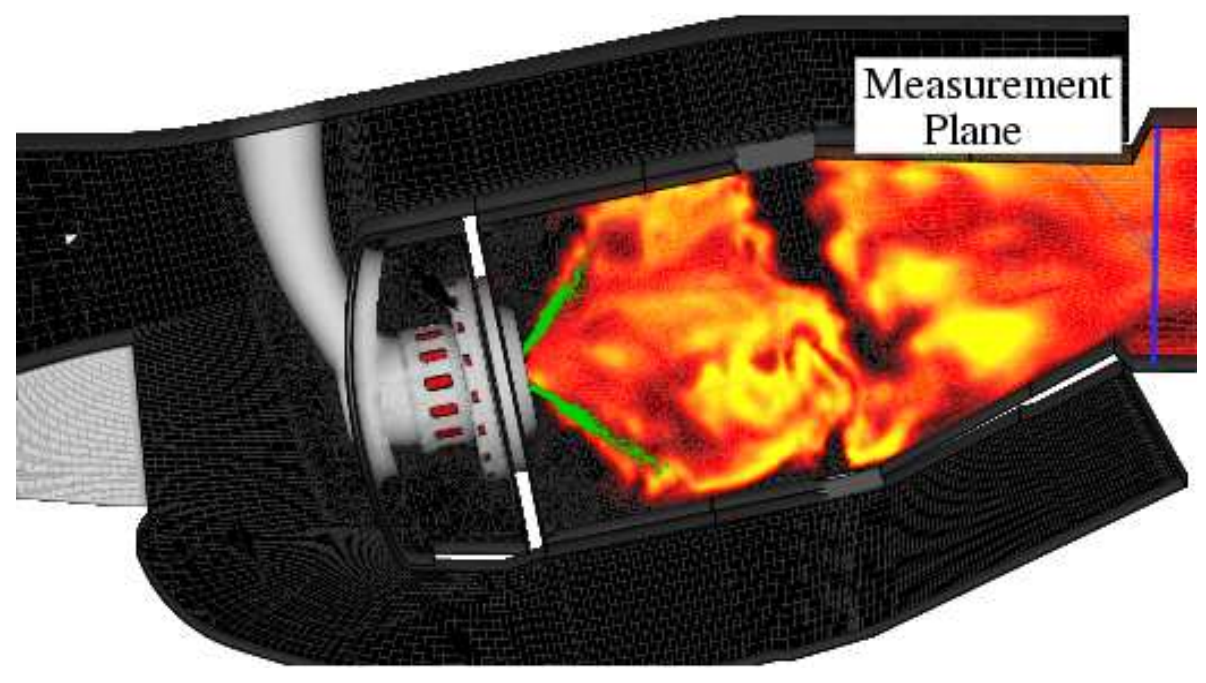

(a)

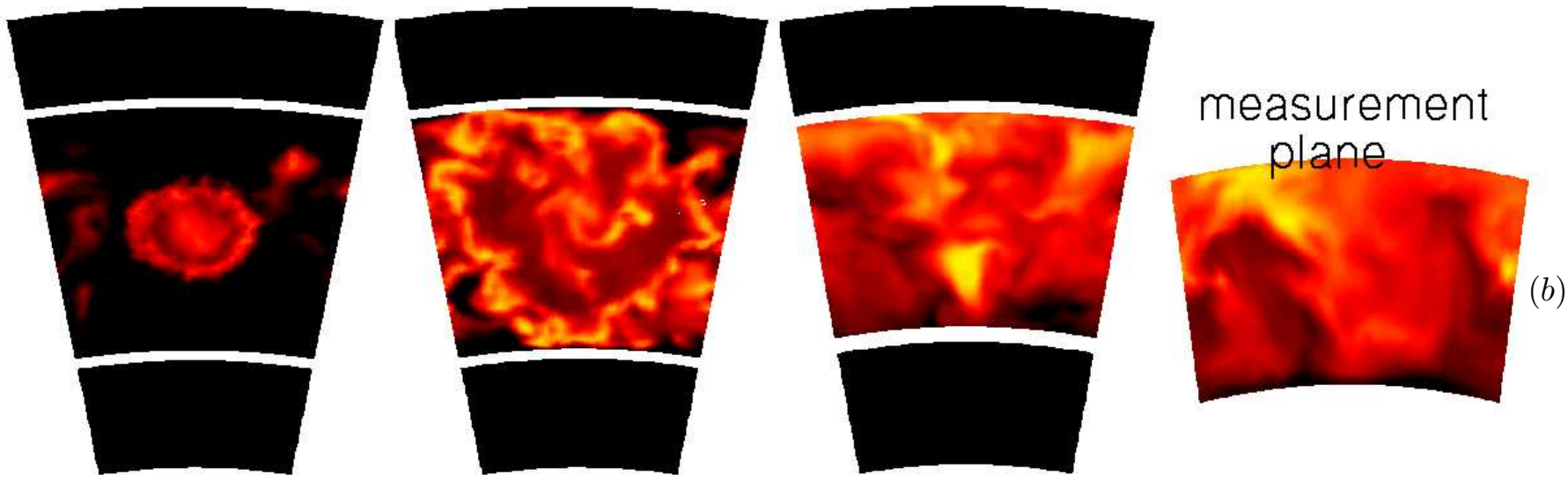

Figure 10: Instantaneous snapshot of temperature in the Pratt \& Whitney combustor obtained from LES: a) symmetry plane with superimposed droplet locations, b) temperature contours at different cross-sections from the injector nozzle, the last plane represents the temperature measurement plane. 


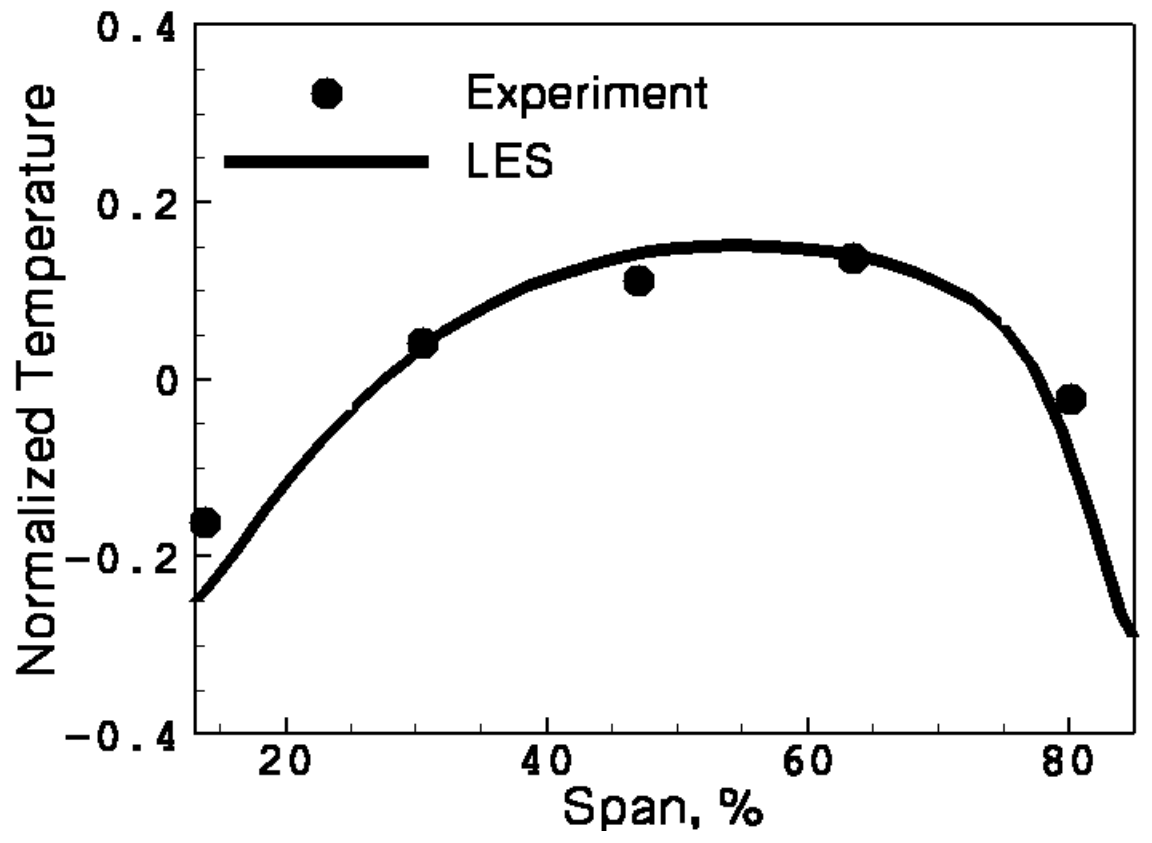

Figure 11: Prediction of mean temperature at the measurement plane of PW combustor. Span represents the normalized vertical distance in the measurement plane. 